\title{
ABOUT RELATIONSHIP BETWEEN THE SIGNAL POWER, NUMBER OF M-QAM POSITIONS AND NOISE IMMUNITY IN BROADBAND WIRELESS ACCESS SYSTEMS
}

\author{
Isa Mammedov, \\ Azerbaijan Technical University, Baku, Azerbaijan, \\ isamamedov@bk.ru \\ Ilham Afandiyev, \\ State Management of Radiofrequencies of Ministry of Transport, \\ Communication and High Technology, Baku, Azerbaijan, \\ ilham.afandiyev@gmail.com
}

DOI: $10.36724 / 2664-066 X-2020-6-2-24-28$

\begin{abstract}
Is studied the effect of measurement error on the accuracy of selecting the modulation type in the transmitter of the base station. The article discusses changes in the statistical characteristics of the measured signal as it passes through the stage of the feedback loop of the system of the LMDS type. Probabilistic characteristics of pilot-signal are determined at the output of this loop. The aim of the work was to find a compromise between the type of modulation and the power of the BS transmitter to maintain the transmission faithfulness within the given limits in the forward channel of an interactive wireless broadband radio access system of the LMDS. In this system a transition is made from one type of modulation to another in order to maintain the noise immunity of the system within the admissible limits. An adaptive controlling method of modulation type makes it possible to obtain an energy gain in the forward channel of the system.

It is useful to determine the type of modulation by measuring the current value of the signal-to-interference ratio at the base station (BS) receiver. For this purpose a pilot signal is transmitted on the reverse channel of the system. This signal, passing through the turbulent medium, undergoes attenuation, and therefore at the input of the BS receiver we have a random process. Moreover, the distribution law of this process can be different and is determined by the communication channel model. The problem consisted in
\end{abstract}

determining the necessary probabilistic characteristics of the output process by using the system parameters and probabilistic characteristics of the input random process. For this purpose the cumulants of the input random process and the random process at the output of the linear inertial system are determined. An expression for the probability density of the random process at the output of a linear inertial system is obtained.

The graphics of the error probability on the energy parameter at 16-QAM and 64-QAM type of modulation are constructed. The influence of the measurement error on the accuracy of the choice of the modulation type in the transmitter of the BS is studied. The limits of the change in the energy parameter are defined graphically, under which the modulation type changes to ensure a given error probability. It is determined, that the system is more critical to the measurement error at high admissible error probability, i.e. at high error probabilities, small errors in the measurement of the signal-to-noise ratio make it necessary to transition to a more noise-immune modulation mode.

KEYWORDS: broadband wireless access system, base station, amplitude-phase modulation, power control, cumulant, adaptive selection of the modulation type

Information about authors

Isa Mammadov, Azerbaijan Technical University, professor, Baku, Azerbaijan

Ilham Afandiyev, State Management of Radiofrequencies of Ministry of Transport, Communication and High Technology, Chief Engineer, Baku, Azerbaijan 


\section{Problem Statement}

In order to provide the population with various types of information and to solve the "last mile" problem, integral telecommunication technologies are used, the varieties of which are wireless broadband radio access systems such as LMDS (Local Multipoint Distribution Systems) and MVDS (Multipoint Video Distribution Systems). These systems are high-speed and are built according to the cellular principle [1].

Various modulation methods have been proposed in LMDS systems [2]. Moreover, in the same base station transmitter (BS), different types of modulation are used, depending on the distance to the subscriber - for distant subscribers, a more noise-resistant type of modulation is used. Such a transition is carried out in order to maintain the system's noise immunity within acceptable limits. Typically, the signal strength decreases as distance to receiver increases. Therefore, in the named system, QPSK modulation is used for distant users, and for near ones it is replaced by M-QAM modulation.

Signal attenuation is random, and the known models of radio signal propagation make it possible to give only conjectural estimates of signal level at the receiving point. Therefore, changing the type of modulation, based on the distance to subscriber station, cannot give the desired result from the point of view of ensuring the necessary transmission fidelity. A quick assessment of the signal level in BS and the method of adaptive selection of the type of modulation and power control over BS transmitter channels may be the most acceptable option to ensure necessary transmission fidelity.

In mobile communication systems, open and closed loop power control methods are used to solve the far-close problem. Also, circuits and algorithms for power regulation in them are drawn up. The reference signal for power control is the pilot signals emitted by the subscriber transmitters.

The aim of this work is to find a compromise between the type of modulation and power of BS transmitter to maintain fidelity of transmission within the specified limits in forward channel of interactive system of wireless broadband radio access of LMDS type.

\section{Controlling the type of modulation in broadband radio access systems}

The power control in the forward channel is of particular importance, since at high power the BS transmitter interferes with other cells and sectors [3]. This adjustment helps reduce forward link interference.

Signal attenuation depends on various factors and the use of a certain type of modulation depending on the distance may not be beneficial due to energy considerations and a decrease in the transmission rate when using the appropriate type of modulation. Therefore, it is of interest to determine the type of modulation by measuring the current value of the signal-to-noise ratio in the BS. Such an adaptive method for controlling the type of modulation will provide an energy gain in the forward channel.

The stated task belongs to the class of systems management. Control is a purposeful impact on the control object to maintain certain parameters within the required limits. System identification, state estimation and control are the main typical tasks of systems control theory. It can be deterministic or stochastic. Typically, external disturbances affect the operation of the control system. If you do not take into account the influence of these interferences, then the control system is considered deterministic. Taking into account the noise and their statistical features, the control system is considered as stochastic [4]. Stochastic control performs the task of optimal control of the system under the influence of noise on the control and measuring units.

Pilot signal emitted by subscriber station, passing through a turbulent medium, undergoes attenuation, and therefore at the input of BS receiver we have a signal:

$$
S(t)=A(t) \cos \left[\omega_{0} t+\varphi_{0}+\varphi(t)\right]
$$

Here $\omega_{0}$ - frequency, $\varphi_{0}$ - initial phase, $A(t)$ - amplitude, $\varphi(t)$ - random phase of the pilot signal at the input of the demodulator. The amplitude and phase of the measured signal are random in this case. Moreover, the laws of their distribution can be different and are determined by the communication channel model [5]. From the given distribution models of the signal value, the distribution of the signal amplitude can be determined. For example, if during slow fading the distribution of the signal value obeys a lognormal law, then the signal amplitude is described by the Rice distribution law [6]. A pilot signal arrives at the detector's input, the amplitude of which obeys one of the above laws.

\section{Change in the statistical parameters of the measured signal when it passes through the control circuit stages}

Pilot signal is fed to the demodulator input. Pilot signal demodulator can be represented by the following block diagram (Fig. 1). The task is to determine required probabilistic characteristics of the output process with known parameters of system and probabilistic characteristics of input random process. In communication theory, filters belong to the class of linear inertial systems. A system is called linear if it obeys of superposition principle. A linear inertial system, at the input of which there is a random process $\xi(t)$, starting from the moment $t=0$, is described by a fluctuation differential equation [4]. Exact solution to this equation obtained for a Gaussian random process.

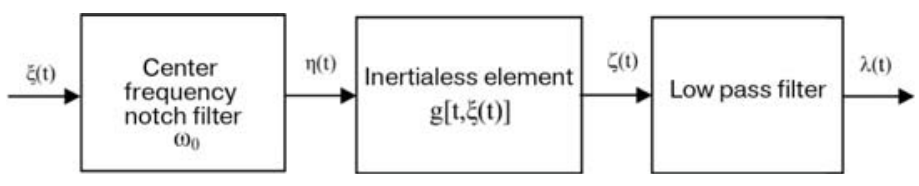

Figure 1. Block diagram of the measuring signal demodulator

We assume that the input random process is stationary in broad sense. Methods for determining the probability density at output of a linear inertial system are known if probability density of input random process is known. Based on the known probability density of input random process, its cumulants are found $[4,6]$ :

$$
\mathfrak{x}_{\xi \mathrm{k}}=\left.\frac{d^{k} \psi_{\xi}(v)}{d v}\right|_{v=0}, \quad k=1,2,3, \ldots \ldots .
$$

Here 


$$
\psi_{\xi}(v)=\ln \theta_{\xi}(v)=\ln \left[\int_{-\infty}^{\infty} w_{\xi}(x) e^{i v x} d x\right],
$$

$w_{\xi}(x)$ - one-dimensional probability density of input random process $\xi(t), \theta_{\xi}(v)-$ its characteristic function.

After calculating the cumulants of the input random process, the transfer function of the linear inertial system is determined. Transfer functions of filters are given in the literature $[4,6]$. Let us assume that the impulse response of this system is expressed by the formula $[4,6]$ :

$$
g(t)=\alpha e^{-\alpha t} . \quad \alpha=\frac{1}{R C}
$$

We calculate the cumulants of output random process $\eta(t)$, for which we use impulse response of this linear system and expression $[4,7]$ :

$$
\frac{\mathfrak{x}_{\eta \mathrm{k}}}{\mathfrak{x}_{\xi \mathrm{k}}}=\int_{0}^{\infty}\left[\int_{0}^{\infty} g(t-u) g(u) d u\right]^{k} d t / \int_{0}^{\infty}[g(t)]^{k} d t .
$$

Integrating in (2), taking into account (1) for values $t \geq 0$ and $\alpha>0$ (which correspond to conditions of our problem), we find:

$$
æ_{\eta k}=\frac{\frac{\alpha^{k}}{4^{k}} \int_{0}^{\infty} e^{-\alpha t} d t}{\frac{\alpha^{k-1}}{k}} \mathfrak{\xi}_{\xi k}=\frac{\mathfrak{x}_{\xi k}}{4^{k}} .
$$

Taking into account the values of the cumulants of the input random process $\xi(t)$ 7] from (3) obtain:

$$
\begin{gathered}
\mathfrak{x}_{\eta 1}=\frac{m_{\xi 1}}{4} ; \quad \mathfrak{x}_{\eta 2}=\frac{m_{\xi 2}-m_{\xi 1}^{2}}{16} ; \\
\mathfrak{x}_{\eta 3}=\frac{3\left(m_{\xi 3}-3 m_{\xi 2} m_{\xi 1}+2 m_{\xi 1}^{3}\right)}{64} ; \\
\mathfrak{x}_{\eta 4}=\frac{m_{\xi 4}-4 m_{\xi 3} m_{\xi 1}-3 m_{\xi 2}^{2}+12 m_{\xi 2} m_{\xi 1}^{2}-6 m_{\xi 1}^{4}}{256}= \\
=\frac{M_{\xi 4}-3 M_{\xi 2}^{2}}{256}
\end{gathered}
$$

where $m_{\xi k}$ and $M_{\xi k}$ - respectively, initial and central moments of distribution of the input random process.

The approximation of the probability density of a random process at the output of a linear inertial system is carried out by the cumulants at the output of this system. We apply the Edgeworth series to approximate the probability density of output random process $[4,6]$. The named series after rearrangement has the form [8]:

$$
\begin{aligned}
& w_{\eta}(y)=\frac{1}{2 \pi} e^{-y^{2} / 2} \times \\
& \times\left[1+\frac{\mathfrak{x}_{\eta 3}}{3}\left(2,7 y^{6}-20 y^{4}+34 y^{2}-6 y-5\right)+\right. \\
& \left.+\frac{\mathfrak{x}_{\eta 4}}{6}\left(4 y^{4}-12 y^{2}+3\right)+\frac{\mathfrak{x}_{\eta 5}}{30}\left(8 y^{5}-40 y^{3}+3 y\right)+\cdots\right]
\end{aligned}
$$

where $H_{n}(y)$ - Hermite polynomials defined by the formulas [4]:

$$
H_{n}(y)=(-1)^{n} e^{y^{2}} \frac{d^{n} e^{-y^{2}}}{d y^{n}}, \quad n=0,1,2, \cdots
$$

For small values of high-order cumulants, we use only the first two terms in (5). Then initial moments of the first, second and third order participate in the approximated probability density of random process at filter output.

Suppose the pilot signal amplitude obeys a Rayleigh distribution. Then for the input random process we define:

$$
\begin{aligned}
& \mathfrak{x}_{\xi 1}=m_{\xi 1}=\sigma_{\xi} \sqrt{\frac{\pi}{2}} ; \mathfrak{x}_{\xi 2}=m_{\xi 2}-m_{\xi 1}^{2}=\frac{4-\pi}{2} \sigma_{\xi}^{2} ; \\
& m_{\xi 2}=2 \sigma_{\xi}^{2} ; \mathfrak{x}_{\xi 3}=M_{\xi 3}=(\pi-3) \sqrt{\frac{\pi}{2}} \sigma_{\xi}^{3} ; \\
& \mathfrak{x}_{\xi 4}=M_{\xi 4}-3 M_{\xi 2}^{2}=0,05 \sigma_{\xi}^{4} .
\end{aligned}
$$

The cumulants of output random process are determined using (4):

$$
\begin{aligned}
& \mathfrak{x}_{\eta 1}=\sigma_{\xi} \sqrt{\frac{\pi}{32}} ; æ_{\eta 2}=\frac{(4-\pi) \sigma_{\xi}^{2}}{32} ; \\
& æ_{\eta 3}=\frac{M_{\xi 3}^{2}}{64}=\frac{(\pi-3)}{64} \sqrt{\frac{\pi}{2}} \sigma_{\xi}^{3} ; æ_{\eta 4}=\frac{0,05 \sigma_{\xi}^{4}}{256} .
\end{aligned}
$$

Then from (5) find:

$$
\begin{aligned}
& w_{\eta}(y) \approx \frac{1}{2 \pi} e^{-y^{2} / 2}\left[1+\frac{(\pi-3)}{64} \sqrt{\frac{\pi}{2}} \sigma_{\xi}^{3} \times\right. \\
& \left.\times\left(2,7 y^{6}-20 y^{4}+34 y^{2}-6 y-5\right)\right]
\end{aligned} .
$$

The resulting expression describes the distribution of pilot signal amplitude at output of feedback loop filter with amplitude fluctuations according to Rayleigh law in the communication channel. This distribution is not symmetric and, for nonsymmetric distributions, Edgeworth series is not the best option. Second term in the middle bracket on the right side of expression (6) is a polynomial. Therefore, it would be convenient here to use Pearson polynomials for approximation. 
To determine probabilistic characteristics of a random process $\zeta(t)$ (Fig. 1), it is necessary to know the characteristics $g[t, \eta(t)]$ of a nonlinear element (detector), where $\eta(t)$ - input, $\zeta(t)$ - output random processes, function $g(\cdot)$ is determined by system parameters. This function is deterministic. We assume that sum of measured signal and fluctuation noise acts at the detector input:

$$
\eta(t)=S(t, \varphi)+n(t),
$$

where $n(t)$ - Gaussian random process with zero mathematical expectation.

The one-dimensional probability density of two independent random processes sum is determined by well-known formula.

Based on the arguments of literature [6], taking into account (7), we find the probability density of a random process at the detector output:

$$
\begin{aligned}
& w_{\zeta}\left(z_{1}, t_{1}\right)=w_{\eta}\left(\frac{z_{1}+y_{0}}{R_{i}}, t\right)+\delta\left(z_{1}\right) \int_{-\infty}^{y_{0}} w_{\eta}\left(y_{1}, t_{1}\right) d y_{1} \\
& z_{1}>0
\end{aligned}
$$

Here $w_{S}\left(x_{1}, t_{1}\right)$ and $w_{n}\left(y_{1}, t_{1}\right)-\mathrm{p}$ obability density of processes $S(t, \varphi)$ and $n(t)$ respectively, $\delta\left(z_{1}\right)$ - lta function.

\section{4. nfluence of measurement accuracy on the system noise immunity}

The literature investigates noise immunity of communication systems with amplitude-phase modulation. The probability of an error under the action of only fluctuation noise was determined [9]:

$$
p_{\text {ou }}\left(h_{\sigma}^{2}\right)=\frac{2\left(1-\frac{1}{\sqrt{M}}\right)}{\log _{2} M} Q\left(\sqrt{\frac{3 \log _{2} M}{2(M-1)} h_{\sigma}^{2}}\right)
$$

where $M$ - number of QAM signal positions, $Q(\cdot)$ - error function, $h_{\sigma}^{2}=\frac{E_{\sigma}}{N_{0}}, N_{0}$ - noise intensity, $E_{\sigma}$ - the energy of one bit, determined by the formula: $E_{\sigma}=T_{\sigma} P_{c}, P_{c}$ - average signal strength, $T_{\sigma}$ - information bit duration: $T_{б}=T_{э c} / \log _{2} M, T_{э с}-$ elementary duration: $T_{э c}=\frac{1}{\Delta F_{c}}, \Delta F_{c}-$ signal spectrum width.

It is shown that real broadband interference can be represented as white Gaussian noise and, at the same time, with some accuracy, the energy parameter can be determined from the noise immunity as the ratio of the average signal energy to the spectral density of the noise and the above interference $h_{\sigma \Sigma}^{2}$ [10]. It is also possible to determine with some accuracy the probability of error under the action of the above-mentioned interference by the formula obtained for signal reception against the background of fluctuation noise. For this, in formula (8) we replace $h_{\sigma}^{2}$ by $h_{\sigma \Sigma}^{2}$

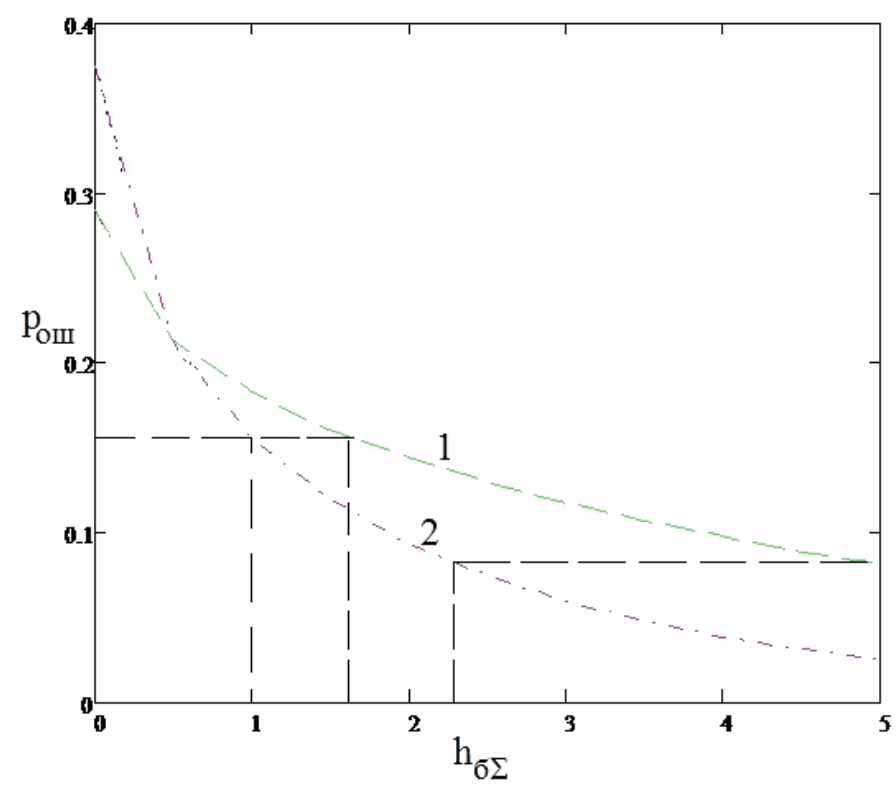

Figure 2. Dependences of the error probability on the energy parameter $h_{\tilde{}}^{2}$ :

1 - if a signal is received when UWB and jitter noise for 64-QAM;

2 - in case of receiving a signal during action UWB interference and fluctuation noise for 16 QAM

We have constructed graphs of the dependence of the error probability $p_{\text {ou }}$ on the energy parameter $h_{\sigma \Sigma}^{2}$ for 16-QAM and 64-QAM in the case of ultra-wideband interference and taking into account fluctuation noise (Fig. 2). It follows from the graphs that, with a high permissible error probability, a small change in the signal-to-noise ratio makes it necessary to switch from one mode to another - a more noise-immune modulation mode. For example, at $p_{\text {ou }}=0.16$ with an increase $h_{\sigma \Sigma}^{2}$ of 1.6 times, it is necessary to change the modulation mode, while at $p_{\text {ou }}=0.08$, such a change must be carried out with an increase $h_{\sigma \Sigma}^{2}$ of 2.27 times. Therefore, in the first case, the measurement system is more sensitive to measurement errors.

\section{Conclusions}

Changing the type of modulation, based on distance to subscriber station, cannot give the desired result in terms of ensuring necessary transmission fidelity. Adaptive choice of type modulation and power control over the BS transmitter channels by realtime estimation of signal level in BS may be the most acceptable option to ensure necessary transmission fidelity.

A pilot signal, passing through a turbulent propagation medium, becomes a random process. For the accuracy of determining power value or signal-to-noise ratio, the change in statistical parameters of this random process at output of main stages in 
closed feedback loop was studied. With a high permissible error probability, small errors in measuring signal-to-noise ratio make it necessary to switch from one mode to another - more noiseimmune modulation mode.

\section{References}

1. http://www.rrl.com.ua/ru/ fo/our/codec/02. 03.06.2016.

2. Hakegard, J.E. Coding and Modulation for LMDS and Analysis of the LMDS Channels. Journal of Research of the National Institute of Standards and Technology, 2000, vol. 105, no 5, pp. 721-734.

3. Bolshov O.A. and Perminov, P.A. Development of feedback channel in the system of wireless broadband access (Cellular Television). Special technique and Communication, 2010, no. 1, pp. 37-45.

4. Levin, B.R. and Shvarts, V. Veroyatnostniye modeli i metodi $v$ sistemakh svyazi i upravleniya [Probabilistic models and methods in communication and control systems]. Moscow: Radio i svyaz. 1985.

5. Evstratov, A.Q. and Pustovoytov, E.L. Calculation of the influence of the interfering radio signal on the receiver of the digital radio communication system under the known distribution laws of fast and slow fading of useful and interfering radio signals. 2014. T-Comm, no.5, pp. 50-55.

6. Levin, B. R. Teoreticheskiye osnovy statisticheskoy radiotekhniki. Kniqa 1 [Theoretical bases of statistical radio engineering. Book 1]. Moscow: Sovetskoye radio. 1974.

7. Mamedov, I.R. and Aliyev, Z.B. Probabilistic description of the amplitude of the pilot signal at the output of a closed feedback loop. Telecommunications, 2012. No. 2, pp. 39-42.

8. Mamedov, I.R. and Aliyev, Z.B. Influence of the accuracy of power adjustment on the noise immunity of the feedback link of mobile communication. T-Comm, 2014, no. 12, pp. 59-61.

9. Cho, K. and Yoon, D. On the General BER Expression of One- and Two-Dimensional Amplitude Modulations. IEEE Trans. on Communications, 2002, vol. 50, July, no. 7, pp. 1074-1080.

10. Aronov, D.A. Estimation of the error probability per bit of transmitted information in the presence of noise and modulated interference. 2007, Telecommunications, no. 11, pp. 56-59. 